\title{
Translationally invariant clusters in coordinate space: higher-order clusters and the Gaussian expansion basis
}

\author{
R F Bishopł, E Buendiat, M F Flynn§ and R Guardiola \\ $\dagger$ Department of Mathematics, UMIST, PO Box 88, Manchester M60 1QD, UK \\ $¥$ Departamento de Fisica Moderna, Universidad de Granada, Facultad de Ciencias, 18071 \\ Granada, Spain \\ § Department of Physics and Liquid Crystal Institute, Kent State University, Kent, OH 44224 , \\ USA \\ I Departamento de Física Atómica y Nuclear, Universidad de Valencia Estudi General, Avda. \\ Dr Moliner 50, 46100-Burjasot, Valencia, Spain
}

Received 15 February 1993

\begin{abstract}
We develop and apply a series of extensible and flexible cluster expansions, tailored specifically to few-body systems. Our analysis begins by recalling previous work which examined the role of the centre-of-mass (CM) motion in the few-body system. We show how the problems generated by the $\mathrm{CM}$ motion generally associated with correlated wavefunctions can be explicitly avoided altogether, and from the outset, by restricting the excitations induced to those which leave the $C M$ undisturbed. This is accomplished by expressing all correlation functions solely in terms of relative coordinates. All such functions are expanded in bases of Gaussians, which are shown to be highly efficient computationally. Applications to the ${ }^{3} \mathrm{H}$ and ${ }^{4} \mathrm{He}$ nuclei are presented. Results show that the method produces both accurate energies and wavefunction properties.
\end{abstract}

\section{Introduction}

In a series of articles [1-7], we have explored the application of the coupled cluster method (CCM) to light nuclei, in particular to the ${ }^{4} \mathrm{He}$ nucleus. So far, our study has been limited to central potentials, thereby allowing a bosonic description of the system, without the additional complications due to Fermi statistics. A major focus has been the reexamination of the centre-of-mass (CM) problem, so ubiquitous in few-body systems such as this. This investigation has led to the development of a new hierarchy of approximations, which while having its origins in the CCM, has evolved into something quite distinct. While the CCM was developed specifically for large or infinite systems, the new hierarchy of calculational schemes, collectively dubbed the translationally invariant cluster (TIC) method, is especially tailored for few-body systems.

One of the main aims of our earlier work has been to develop a formalism in which one can systematically include the effects of higher-order correlations in a manner which both exactly incorporates at all stages rotational and translational invariance and is computationally efficient. It is also important to realize from the outset that at every level of implementation the resulting TIC method (TICM) is variational in the sense of providing an upper bound to the energy eigenvalues. In the preceding paper in this series [7] we focused attention on the lowest-order TIC approximation which keeps only pair correlations. More specifically, we have obtained the optimal two-body correlation functions at this level 
of approximation for the ${ }^{4} \mathrm{He}$ nucleus interacting via a number of two-body internucleonic potentials. These optimal correlations were obtained via a functional (Euler-Lagrange) minimization of the approximate energy functional. By comparison, we have been able to demonstrate that virtually identical energy eigenvalues and eigenfunctions may be obtained using a two-body correlation function parametrized as a linear combination of a very few $(\leqslant 5)$ Gaussians.

In view of the extremely high accuracy attainable by such very small bases at this lowest-order (two-body) level, we are now encouraged to extend their use to incorporate in a systematic fashion the effects of triplet and higher-order correlations, as alluded to in an earlier paper [7]. As we shall see, this improvement of the wavefunction is in fact carried out quite easily, in spite of earlier claims that it would probably prove to be infeasible [8].

The use of Gaussian bases for many-body calculations is not new. Indeed, their use within quantum chemistry, for example, dates back over more than 40 years [9]. It is worth remarking that their use is, in fact, absolutely crucial for numerical ab initio quantum chemistry. Without them even the most powerful modern computers would be useless for typical modern molecular computations. As Wilson [10] has forcefully pointed out recently, the single most important algorithmic advance for modern ab initio computational quantum chemistry was the replacement of numerical grids by analytic basis functions [11]. The analytical functions now used almost exclusively are Gaussians centred on atomic nuclei, coupled with polynomial prefactors to represent spherical harmonics. The high precision attained for the quite large and complicated molecules now studied is in large part due to the fact that even triplet correlations can be calculated accurately, due to the remarkably small size of the so-called contracted Gaussian basis sets (i.e. linear combinations of Gaussians of different widths) needed.

In quantum chemistry, the Gaussian bases are used initially at the atomic level to represent the single-electron Hartree-Fock (HF) wavefunctions. Wilson [10] hypothesizes that when approximately solving the self-consistent $\mathrm{HF}$ field equations in terms of a contracted Gaussian basis, the convergence to the basis set limit is exponentially fast (probably as $\mathrm{e}^{-c N^{1 / 6}}$, where $c$ is a positive constant, and $N$ is the number of Gaussians). By contrast, methods for solving partial differential (or integrodifferential) equations of the HF type on grids converge much more slowly (typically as $N^{-2}$ where $N$ is the number of grid points). Similar much slower convergence is also typical in such complete orthogonal bases as HO wavefunctions (as observed, for example, in [1] for the present problem at the $\operatorname{TIC}\left(C_{2}\right)$ (two-body) level described below).

The general idea of also expressing correlations directly in terms of contracted Gaussian functions of the interparticle spacing is also quite old, and has its origins in what the quantum chemists call the Gaussian geminal basis [12-14]. Recent applications of Gaussian geminals within the context of many-body perturbation theory to calculate manymbody correlation effects beyond the $\mathrm{HF}$ approximation include the work of Szalewicz and co-workers $[15,16]$.

Wilson [10] has recently urged the use of Gaussian basis functions and other techniques from computational chemistry, as an alternative to standard methodology within lattice gauge theory. In the same spirit, we demonstrate here that Gaussian bases are ideally suited to the treatment of few-body problems in nuclear physics (and presumably beyond) as formulated within our own TICM treatment. In particular, we extend and utilize the geminal basis concept to the triplet and quadruplet level.

In section 2 we present in detail the translationally invariant cluster method, as well as the various levels of approximations. This is followed in section 3 by the application of Gaussian expansions to the TICM. Results for energies and wavefunction properties for ${ }^{3} \mathrm{H}$ and ${ }^{4} \mathrm{He}$ are shown in section 4 , along with comparisons with other methods. Finally, 
section 5 discusses the method, and suggests future extensions of it.

\section{Cluster decompositions: an outline of the TIC method}

As stated above, the point of departure from which the TIC method (TICM) sprang is the treatment of the $\mathrm{CM}$ notion problem. Both the CCM and TICM start by writing the wavefunction in the quite general form

$$
\Psi\left(\{i\}_{N}\right)=C\left(\{i\}_{N}\right) \Phi\left(\{i\}_{N}\right)=C\left(\{i\}_{N}\right) \Phi_{\text {int }}\left(\left\{i_{\text {int }}\right\}_{N}\right) \Phi_{\mathrm{CM}}(R)
$$

where $\{i\}_{N}$ represents some set of state variables for the $N$ particles in the system, $C\left(\{i\}_{N}\right)$ is some (at this point quite general) correlation operator, and we have assumed that the model state $\Phi$ can be decomposed into a product of two independent pieces, one depending only on some set of intrinsic coordinates $\left\{i_{\text {int }}\right\}_{N}$, and the other depending only on the $\mathrm{CM}$ coordinate vector $\boldsymbol{R}=\frac{1}{N} \sum_{i} \boldsymbol{r}_{i}$. The model state $\Phi$ is assigned the role of imposing the proper overall symmetry, as well as introducing some sort of global spatial localization. In applications of the traditional CCM to such light systems [17], the correlations induced in the system must, by the nature of the wavefunction decomposition used, produce excitations in $\Phi_{\mathrm{CM}}$. In fact, the subsequent disentanglement of the $\mathrm{CM}$ excitation is by no means trivial $[17,18]$. In [1], we showed how this can be avoided, while still maintaining much of the basic CCM formalism, by restricting the excitations produced by $C\left(\{i\}_{N}\right)$ to those which explicitly commute with $\Phi_{\mathrm{CM}}$, thereby producing as an important by-product a considerable reduction in the amount of computational work required. Unfortunately, the method still suffers from the all too familiar slow convergence of the underlying harmonic oscillator (HO) basis.

In subsequent work [2-7], we demonstrated how the essential features of the TICM could be expressed much more generally and efficiently by recasting the entire formalism directly in coordinate space. More concretely, we rewrite (1) as

$$
\Psi\left(\left\{r_{i j}\right\}_{N}, R\right)=C\left(\left\{r_{i j}\right\}_{N}\right) \Phi_{\text {int }}\left(\left\{r_{i j}\right\}_{N}\right) \Phi_{\mathrm{CM}}(R)
$$

where $\left\{r_{i j}\right\}_{N}$ represents the set of relative coordinates of the $N$-particle system. Now, as the correlations induced by $C$ depend explicitly only on the relative coordinates, they clearly leave the $\mathrm{CM}$ in its original state $\Phi_{\mathrm{CM}}(\boldsymbol{R})$. Further progress is made after assuming an additive decomposition of $C\left(\left\{r_{i j}\right\}_{N}\right)$

$C\left(\left\{\boldsymbol{r}_{i j}\right\}_{N}\right)=1+\sum_{i<j}^{N} C_{2}\left(r_{i j}\right)+\sum_{i<j<k}^{N} C_{3}\left(r_{i j}, r_{i k}, r_{j k}\right)+\sum_{i<j<k<i}^{N} C_{4}\left(\left\{r_{m n}\right\}_{4}\right)+\cdots$

We emphasize that the structure of the $C_{n}\left(\left\{r_{i j}\right\}_{n}\right)$ is at this point completely arbitrary, except that we require symmetry with respect to the $\left\{r_{i j}\right\}_{n}$ in order to preserve the overall symmetry imposed by $\Phi_{\text {int }}$. This is illustrated below, where a variety of functional forms inspired by various many- and few-body methods, are applied to the bosonic ${ }^{4} \mathrm{He}$ nucleus. Note also that the intrinsic variables chosen are the set of relative coordinates $\left\{r_{i j}\right\}_{n}$, rather than the more traditional choice of Jacobi coordinates. It is trivial to show that these form a complete translationally and rotationally invariant description of the $n$-body cluster provided $n \leqslant 4$, and thus are well suited for the type of few-body system of interest here. 
This non-specificity of the sub-cluster functions can be exploited to tailor the correlations to the specific system at hand, as well as to include true high-order correlations in some approximate way. We demonstrate this with a comparison of the TICM with the $C C$ and configuration-interaction $(\mathrm{Cl})$ methods. For bosonic systems, the former is written as an exponential of correlation operators

$$
\Psi_{C C}=e^{S_{1}+S_{2}+S_{3}+S_{4}+\cdots} \Phi_{0}
$$

where each $S_{n}$ is written as a sum of products, each involving precisely $n$ destruction and $n$ creation operators:

$$
S_{n}=\sum_{k_{1}, \cdots, k_{n}} S_{k_{1} \cdots k_{n}}^{(n)} a_{k_{1}}^{\dagger} \cdots a_{k_{n}}^{\dagger}\left(a_{0}\right)^{n}
$$

The $a_{0}\left(a_{k_{i}}^{\dagger}\right)$ destroy (create) particles inside (outside) the Bose condensate defined by the $n$-body model state $\Phi_{0}$, where $\left|\Phi_{0}\right\rangle=(n !)^{-1 / 2}\left(a_{0}^{\dagger}\right)^{n} \mid$ vac $\rangle$. The $\mathrm{Cl}$ method is simpler, being just a linear sum:

$$
\Psi_{\mathrm{Cl}}=\left(1+C_{1}+C_{2}+C_{3}+C_{4}+\cdots\right) \Phi_{0} .
$$

This has the appearance of being identical to (2), but in fact is quite different, as the $C_{i}$ here are written in the very specific form of (5). In the $\mathrm{CC}$ and $\mathrm{Cl}$ methods, this is done to produce correlation operators which excite $n$, and only $n$ particles. Unfortunately, as mentioned above and discussed at length in [1], this is precisely what causes problems with the $\mathrm{CM}$ motion for light few-body systems, since a correlation of this form must violate translational invariance.

However, this does not mean that we cannot borrow lessons learned from these methods. For example, expansion of (4) and omission of vanishing terms leads to a wavefunction for the four-body problem of the form

$\Psi_{\mathrm{CC}}=\left(1+\sum_{n=1}^{4} \frac{1}{n !} S_{1}^{n}+S_{2}+S_{1} S_{2}+\frac{1}{2 !} S_{1}^{2} S_{2}+S_{1} S_{3}+\frac{1}{2 !} S_{2}^{2}+S_{3}+S_{4}\right) \Phi_{0}$.

From past experience, it is known that an important type of four-body correlation is generated by the $S_{2}^{2}$ term in the above equation. Below, we construct a TIC $C_{4}$ precisely along these lines.

The next stage in the development requires that we must choose some specific functional form for the $C_{n}\left(\left\{r_{i j}\right\}_{n}\right)$, and this is first illustrated for the simplest function, $C_{2}\left(r_{i j}\right)$. This is particularly simple in this case: it is simply some arbitrary function of the single variable $r_{i j}$. In fact, the arbitrariness of the $C_{n}\left(\left\{r_{i j}\right\}_{n}\right)$ functions permits a type of simplification which we shall use quite frequently: we may absorb the factor one appearing in (3), producing

$$
\Psi_{\mathrm{T}\left(\mathrm{C}\left(C_{z}\right)\right.}=\left(\sum_{i<j}^{N} C_{2}\left(r_{i j}\right)\right) \Phi_{\mathrm{int}}\left(\left\{r_{m n}\right\}\right) \Phi_{\mathrm{CM}}(\boldsymbol{R})
$$

The notation $\operatorname{TIC}\left(C_{2}\right)$ signifies the translationally invariant cluster method truncated at the $C_{2}$ level. Note the strong similarity between this wavefunction and (6), truncated at the two-body (Sub(2)) order, though as mentioned above, unlike a pure $\mathrm{CI}$ wavefunction, the correlations included here are not of a pure two-body nature, as they would be for both the 
CI and CC methods. As explained in detail in [1], this is required in order to maintain the translational and rotational invariance of the wavefunction.

We now turn to improvements on the $\operatorname{TIC}\left(C_{2}\right)$ wavefunction. As we already have a complete translationally and rotationally invariant $C_{2}\left(r_{i j}\right)$, we must turn to the higherorder clusters. In principle, the next cluster is $C_{3}\left(r_{i j}, r_{i k}, r_{j k}\right)$, but for mainly historical reasons we shall first consider a partial inclusion of $C_{4}\left(\left\{r_{m n}\right\}_{4}\right)$. While we will examine below a complete description, we first try a much simpler functional form, again taking our inspiration from a many-body theory, this time the $\mathrm{CCM}$, where the next-order term in (7) induces separate correlations between two independent pairs of particles. Thus, the corresponding TIC wavefunction would be of the following form for a four-body system [1]:

$$
\Psi_{\mathrm{TIC}\left(C_{2}^{2}\right)}=\left(1+\sum_{i<j}^{4} C_{2}\left(r_{i j}\right)+\frac{1}{2} \sum_{i<j}^{4} C_{2}\left(r_{i j}\right) C_{2}\left(r_{k l}\right)\right) \Phi_{\mathrm{int}}\left(\left\{r_{m n}\right\}\right) \Phi_{\mathrm{CM}}(R)
$$

where in the last term the indices $k$ and $l$ indicate the remaining two particles after particles $i$ and $j$ have been selected. In fact, within the TiCM, it is actually easier to use the more general functional form $C_{2}^{(2)}\left(r_{i j}, r_{k l}\right)$ in place of the quadratic term in (9). (The superscript 2 is placed in parentheses to remind us that it does not indicate the square of the operator.) This is not precisely true either. Just as above we could absorb the constant term one in (8), once again the complete arbitrariness of the function $C_{2}^{(2)}\left(r_{i j}, r_{k l}\right)$ can be utilized to absorb all of the lower-order terms. Thus, the wavefunction defining the $\operatorname{TIC}\left(C_{2}^{(2)}\right)$ approximation is

$$
\Psi_{\mathrm{TIC}\left(C_{2}^{(2)}\right)}=\left(\sum_{i<j}^{4} C_{2}^{(2)}\left(r_{i j}, r_{k l}\right)\right) \Phi_{\text {int }}\left(\left\{r_{m n}\right\}\right) \Phi_{\mathrm{CM}}(R)
$$

We now tum to the three-body clusters. Here, we also have a great deal of flexibility in choosing the functional form of $C_{3}\left(r_{i j}, r_{i k}, r_{j k}\right)$. While we can (and do) use directly this function of three variables, a simpler possibility is to choose some function of only one variable. This time taking inspiration from a few-body technique, we express this function as

$$
C_{3}\left(r_{i j}, r_{i k}, r_{j k}\right) \rightarrow C_{3}^{\mathrm{S}}\left(h_{3}\right)
$$

where $h_{3}$ is equal to the three-body hyperspherical radius $h_{3} \equiv\left(r_{12}^{2}+r_{13}^{2}+r_{23}^{2}\right)^{1 / 2}$, precisely as defined in the hyperspherical harmonics method [19].

The two functional forms for the three-body cluster define the $\operatorname{TiC}\left(C_{3}\right)$ approximations, referred to as full (F) and simple (S), respectively. If combined with the $\operatorname{TiC}\left(C_{2}^{(2)}\right)$ function of (10), they produce the $\operatorname{TiC}\left(C_{2}^{(2)}+C_{3}\right)$ approximation, otherwise, they result in $\operatorname{TIC}\left(C_{2}+C_{3}\right)$.

Before continuing, a few words are in order about the flexibility that the TIC methodology allows. Beginning with the lowest-order $\operatorname{TIC}\left(C_{2}\right)$, we were free to absorb the constant term into our definition of $C_{2}\left(r_{i j}\right)$. At the $\operatorname{TIC}\left(C_{2}^{(2)}\right)$ level, we absorbed all of the lower-order pieces into the highest-order term. Here, if we use the form $C_{3}^{\mathrm{F}}\left(r_{i j}, r_{i k}, r_{j k}\right)$, we do the same, resulting in the $\operatorname{TIC}\left(C_{3}^{\mathrm{F}}\right)$ wavefunction. For the $C_{3}^{\mathrm{S}}\left(r_{i j}, r_{i k}, r_{j k}\right)$ term, however, this cannot be done, as the partial description of the three-body cluster provided is not sufficient to render redundant the lower-order two-body terms. Thus, we maintain the lower-order term, and use the TIC $\left(C_{2}+C_{3}^{S}\right)$. This freedom to absorb the lower-order cluster terms comes about because the full function $C_{n}\left(\left\{r_{i j}\right\}_{n}\right)$ completely describes the $n$-body cluster, and thus 
is not limited to inducing only $n \mathrm{p}-n \mathrm{~h}$ excitations, as are the $\mathrm{CC}$ and $\mathrm{Cl}$ correlation operators of the form (5). Of course, for an infinite-body system, there are very good reasons indeed for imposing this limitation, but this is not a problem for the few-body systems considered here.

We now return to the four-body cluster term. To date, two approximation schemes involving this level of clustering, in addition to the $\operatorname{TIC}\left(C_{2}^{(2)}\right)$ discussed above, have been considered. The simplest is the exact analogue of the $\operatorname{TrC}\left(C_{3}^{S}\right)$, with the $C_{4}^{S}\left(\left\{r_{m n}\right\}_{4}\right)$ depending only on the four-particle hyper-radius, $h_{4}=\left(\sum_{i<j}^{4} r_{i j}^{2}\right)^{1 / 2}$. Alternatively, we can attempt a six-variable function, which in principle would provide a complete description of the four-particle systems considered here. As seen below, this latter representation presents some difficulties in practical applications. We present results below for the $\operatorname{TIC}\left(C_{2}^{(2)}+C_{3}^{\mathrm{F}}+C_{4}^{\mathrm{S}}\right)$ approximation in a notation which should by now be self-evident, as well as for some simpler approximations.

\section{Cluster decompositions: Gaussian expansions}

We now turn our attention to determining the functions described in the previous section. Once again, we start with the lowest-level approximation, $\operatorname{TIC}\left(C_{2}\right)$, and solve for the twobody function, $C_{2}(r)$. Again unlike the standard ${ } C$ method, for example, we choose to do this variationally, i.e. we solve for $C_{2}(r)$, along with the associated energy, via some minimization technique. To date, this has been done in two ways. In [7], the optimal EulerLagrange solution for the $\operatorname{TIC}\left(C_{2}\right)$ wavefunction (there referred to as $\operatorname{TICI}(2)$ ) is presented in great detail. In this paper, we focus on an alternative technique, where we expand in the overcomplete basis of Gaussians

$$
C_{2}(r)=\sum_{i}^{i_{\operatorname{mex}}} A_{i} \mathrm{e}^{-b_{1} r^{2}}
$$

were we have indicated that in practice the sum must be truncated at some finite level. With this expression, the linear parameters $\left\{A_{i}\right\}$ are determined through the solution of a generalized eigenvalue equation, with the matrix elements being nonlinear functions of the parameters $\left\{b_{i}\right\}$ as well as any variational parameters in the model states $\Phi_{\mathrm{CM}}(\boldsymbol{R})$ and $\Phi_{\text {int }}\left(\left\{r_{i}\right\}\right)$. This ansatz has proved to be very efficient computationally. In addition, and more importantly, it produces essentially the same energy and wavefunction as the optimal results for all potentials considered so far [7], even with bases of very small size $\left(i_{\max } \leqslant 5\right)$.

A perhaps non-trivial point concerning (12) is that the nonlinear parameters $\left\{b_{i}\right\}$ are not required to be positive semi-definite, as one might at first expect. All that is required is that the overall wavefunction (including the global multiplicative factor $\Phi_{\mathrm{CM}}(R)$ ) be normalizable. This allows the use of correlation functions which rise (or drop) to infinity at large distances. In fact, it turns out that such negative values for the $\left\{b_{i}\right\}$ are very important in achieving good energy values. An example of such a non-bound correlation function is shown in figure 4 of [7]. The points made here also apply to the higher-order correlation functions presented below.

We turn now to the higher-order clusters, beginning with the $\operatorname{TiC}\left(C_{2}^{(2)}\right)$ ansatz of $(10)$. Once again, Gaussians are used to express this function of two variables, via the double expansion

$$
C_{2}^{(2)}\left(r, r^{\prime}\right)=\sum_{m \leqslant n}^{m_{\text {max }}, n_{\max }} A_{m n} \mathcal{S}\left(e^{-\left(b_{m} r^{2}+b_{n} r^{2}\right)}\right)
$$


The symbol $\mathcal{S}$ indicates that symmetrization with respect to particle indices must be performed. As before, all the linear parameters $\left\{A_{m n}\right\}$ can be determined efficiently through a generalized eigenvalue equation, and only the nonlinear parameters $\left\{b_{m}\right\}$, along with any other variational parameters of the model states $\Phi_{\text {int }}$ and $\Phi_{\mathrm{CM}}$, need be found via some minimization algorithm. We note that the term linear in $C_{2}$ in (9) may be explicitly recovered by setting one of the parameters $\left\{b_{n}\right\}$ to zero.

Following the outline of the previous section, we next examine the three-body terms. The simple functional form of (11) can again be expanded as a single Gaussian expansion, and thus is hardly more difficult to implement than the lowest-order wavefunction, $\operatorname{TIC}\left(C_{2}\right)$. With a little more effort, a complete translationally and rotationally invariant description of the three-body cluster can be achieved via the triple Gaussian expansion

$$
C_{3}^{\mathrm{F}}\left(r_{12}, r_{13}, r_{23}\right)=\sum_{m \leqslant n \leqslant p} A_{m n p} \mathcal{S}\left(\mathrm{e}^{-\left(b_{m} r_{12}^{2}+b_{n} r_{13}^{2}+b_{p} r_{23}^{2}\right)}\right)
$$

As with $\operatorname{TIC}\left(C_{2}^{(2)}\right)$, the Gaussian terms must be symmetrized.

The same pattern is followed for the four-body cluster expansions. The single-variable function $C_{4}^{\mathrm{S}}\left(\left\{r_{m n}\right\}_{4}\right)$ is expressible via a simple Gaussian expansion, whereas the full $C_{4}$ requires a six-fold Gaussian expansion, which in principle provides a complete description of the four-particle systems considered here

$$
C_{4}^{\mathrm{F}}\left(\left\{r_{i j}\right\}_{4}\right)=\sum_{n_{1} \leqslant \ldots \leqslant n_{6}} \mathcal{S}\left(A_{n_{1} \ldots n_{6}} \mathrm{e}^{-\left(b_{n_{1}} r_{12}^{2}+\ldots+b_{n_{6}} r_{34}^{2}\right)}\right)
$$

\section{Results}

\subsection{Energy results for ${ }^{3} \mathrm{H}$ and ${ }^{4} \mathrm{He}$}

In this section, results are presented for the two boson-like nuclei, namely ${ }^{3} \mathrm{H}$ and ${ }^{4} \mathrm{He}$ described via state-independent central potentials. Included are the soft infinite-core MT-V potential of Malfiet and Tjon [20], the spin-averaged s3 potential of Afnan and Tang [21], and the phenomenological potentials $\mathrm{V} 7$ of Volkov [22] and B1 of Brink and Boeker [23]. The first two are fitted to the low-energy phase-shift data, and so may be considered semi-realistic. As there is some variety in the parameters used in the MT-V potential, we refer the interested reader to [1] for the explicit form used. For all calculations, we set $\hbar^{2} / m=41.5 \mathrm{MeV} \mathrm{fm}^{2}$. For both systems, we make the natural choice of the harmonic oscillator (HO) ground-state wavefunction $\phi_{0}\left(r ; \alpha^{-1 / 2}\right)$ for the model state $\Phi_{\text {int }}$ of (2)

$$
\Phi_{\mathrm{int}}\left(\left\{r_{m n}\right\}, \alpha\right)=\prod_{i<j=1}^{N} \phi_{0}\left(r_{i j} ; \alpha N^{-1 / 2}\right) \quad \Phi_{\mathrm{CM}}(\boldsymbol{R})=\phi_{0}\left(\boldsymbol{R}, \alpha N^{1 / 2}\right)
$$

where $\alpha$ is the inverse length scale, which is itself treated as a variational parameter.

In table 1 , results are shown for the triton, described with the MT-V potential, calculated via the $\operatorname{TiC}\left(C_{3}^{\mathrm{F}}\right)$ wavefunction. Note that, for this (or any) three-body system interacting via a pairwise central potential, a wavefunction using (14) provides a complete description. This is clearly demonstrated in the results shown, with the energy obtained being essentially the same as from the also-exact Fadeev method [24], as well as that of the Green function 
Table 1. Ground-state enengy values for the ${ }^{3} \mathrm{H}$ nucleus described by the MT-Y potential. Shown are results for the $\operatorname{Tr}\left(C_{3}^{F}\right)$ method presented in the text, configuration-space Fadeey theory (CSF) [24], the Green function Monte Carlo (GFMC) [25], the variational Jastrow Monte Carlo (VMC) [26], and the ATMS method [27].

\begin{tabular}{ll}
\hline Method & Energy (MeV) \\
\hline $\operatorname{TIC}\left(C_{3}^{\mathrm{F}}\right)$ & -8.22 \\
CSF & -8.25 \\
GFMC & $-8.26 \pm 0.01$ \\
VIMC & $-8.22 \pm 0.02$ \\
ATMS & -8.26 \\
\hline
\end{tabular}

Table 2. Resuits for the ground-state energy of the ${ }^{4} \mathrm{He}$ nucleus (expressed in $\mathrm{MeV}$ for various potentials, calculated in various translationaliy invariant cluster (TIC) approximations. Also shown are results from the independent-particle harmonic oscillator (HO) variational calculation and the diffusion Monte Carlo (DMC) results of [28].

\begin{tabular}{|c|c|c|c|c|}
\hline \multirow[t]{2}{*}{ Method } & \multicolumn{4}{|c|}{ Potential } \\
\hline & BI & v7 & s3 & $M T-V$ \\
\hline но & -28.2 & -27.3 & -5.9 & -6.4 \\
\hline $\operatorname{\pi c}\left(C_{2}\right)$ & -37.82 & -28.66 & -25.37 & -29.41 \\
\hline$\pi C\left(C_{2}^{(2)}\right)$ & -37.90 & -28.68 & -25.56 & -29.59 \\
\hline $\operatorname{TrC}\left(C_{2}+C_{3}^{S}\right)$ & -38.08 & -28.68 & -26.67 & -30.71 \\
\hline $\begin{array}{l}\operatorname{TIC}\left(C_{2}+C_{3}^{5 \mathrm{~s}}+C_{4}^{\mathrm{s}}\right) \\
\operatorname{TIC}\left(C_{3}^{\mathrm{s}}\right)\end{array}$ & -38.26 & -28.72 & -27.05 & $\begin{array}{l}-30.72 \\
-31.04\end{array}$ \\
\hline $\begin{array}{l}\operatorname{\pi TC}\left(C_{2}^{(2)}+C_{3}^{5}\right) \\
\pi \mathrm{TC}\left(C_{2}^{(2)}+C_{3}^{5}+C_{4}^{\mathrm{S}}\right)\end{array}$ & -38.17 & -28.69 & -26.83 & $\begin{array}{l}-30.91 \\
-30.98\end{array}$ \\
\hline $\begin{array}{l}\pi \mathrm{C}\left(C_{2}^{(2)}+C_{3}^{\mathrm{F}}\right) \\
\operatorname{\pi C}\left(C_{2}^{(2)}+C_{3}^{\mathrm{F}}+C_{4}^{\mathrm{S}}\right)\end{array}$ & -38.30 & -28.73 & -27.21 & $\begin{array}{l}-31.24 \\
-31.24\end{array}$ \\
\hline DMC & $-38.32 \pm 0.01$ & $-28.76 \pm 0.01$ & $-27.35 \pm 0.02$ & $-31.32 \pm 0.02$ \\
\hline
\end{tabular}

Monte Carlo (GFMC) method [25]. We also show the corresponding results obtained with the variational Jastrow Monte Carlo method [26] and the ATMS method [27].

In table 2 we show values for the ground-state energies for the ${ }^{4} \mathrm{He}$ nucleus described by various two-body central potentials. For comparison, we show the exact (within the quoted statistical errors) diffusion Monte Carlo (DMC) results from [28], as well as the zeroth-order (HO) results with $C_{2}(r)$ set equal to one (i.e. minimization with respect to the harmonic oscillator parameter $\alpha$ only).

All in all, the results are impressive. Furthermore, we remind the reader that all of our TIC energies are strict variational upper bounds. Even for the very simple $\operatorname{TIC}\left(C_{2}\right)$ wavefunction we are missing less than $2 \mathrm{MeV}$ for the worst case. Adding the CCM Sub(2)like excitation to produce $\operatorname{TiC}\left(C_{2}^{(2)}\right)$ helps somewhat, but a much bigger improvement in the energy comes with the inclusion of the three-body correlations. It is very encouraging to see that even the simple $\operatorname{TIC}\left(C_{2}+C_{3}^{\mathrm{S}}\right)$ method which employs three-body correlations of the form given by (11), and which is hardly more difficult to implement than the $\operatorname{TIC}\left(C_{2}\right)$ method, produces most of the energy gained by inclusion of the full three-body translationally and rotationally invariant correlation. The best results shown, using the $\operatorname{TrC}\left(C_{2}^{(2)}+C_{3}^{\mathrm{F}}\right)$ wavefunction, are indistinguishable from the DMC results for the Bl potential, and for the $\mathrm{V} 7, \mathrm{~S} 3$ and MT-V potentials, are $0.01,0.10$ and $0.04 \mathrm{MeV}$ outside of the $2 \sigma$ confidence range, respectively. It is clear that the three-body correlations are important. 
In order to investigate the effect of the four-body correlations beyond the level incorporated by the operators $C_{2}^{(2)}$ and $C_{4}^{\mathrm{F}}$, we have also performed some calculations using the $C_{4}^{S}$ of (15). Unfortunately, the sixfold Gaussian expansion is not very practical, since the number of integrals required for the evaluation of a matrix element grows very rapidly with the truncation index. For example, keeping two, three and four Gaussians in each relative coordinate variable leads respectively to 2510,283305 and 8582176 integrals. The corresponding ground-state energies for the ${ }^{4} \mathrm{He}$ nucleus with the MT-V potential are, respectively, $-29.0,-30.8$ and $-31.2 \mathrm{MeV}$. Clearly, with one or two Gaussians more in each of the six expansions, the energy would converge rapidly. Nevertheless, we reiterate that this is not recommended as a practical calculational technique for including four-body correlations.

A few words are warranted concerning the efficiency of the TICM. First, the convergence of the Gaussian expansion is very rapid. Only a few terms are needed in all cases to achieve almost the entire energy for the chosen wavefunction, and generally complete convergence to the level of accuracy of a few hundredths of $1 \mathrm{MeV}$ in binding energy is achieved with four to (in extreme cases) eight terms in each of the separate summations. In addition, for each of the potentials considered here, which are either sums of Gaussians or Yukawians, all integrals for all TIC energy results have been evaluated analytically. This essentially eliminates errors, as well as speeds up the necessary numerical calculations. We should mention, however, that while beneficial it is not necessary for the potential to have this form. Some preliminary work for potentials of other forms has been carried out and will be reported later. As long as one does not introduce non-central dependence on the coordinatespace variables, at most one-dimensional integrals of the form $\int_{0}^{\infty} V(x) \mathrm{e}^{-\alpha x^{2}} \mathrm{~d} x$ need be evaluated numerically, and in general even these can be evaluated once and tabulated, leaving then only a table look-up to perform during the actual calculation.

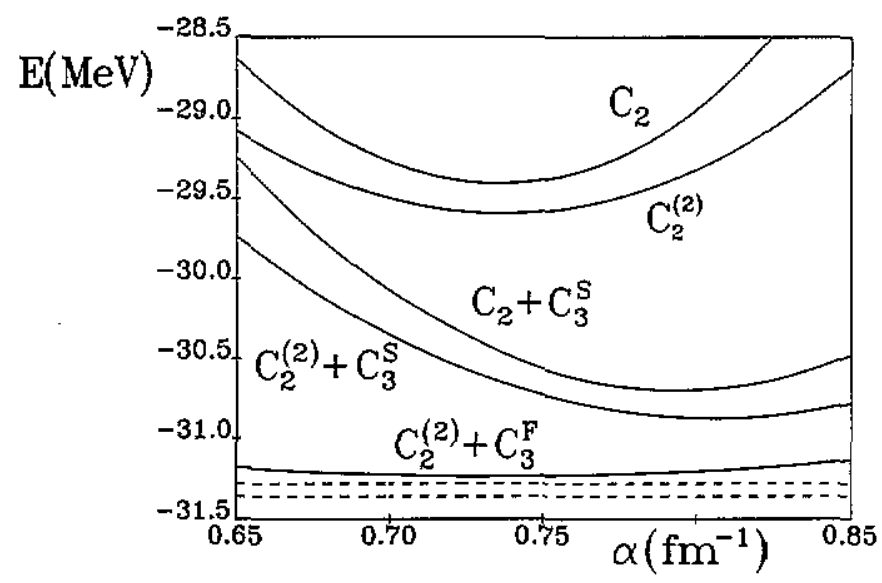

Figure 1. Dependence of the ground-state energy for the MT-V potential on the variational harmonic oscillator parameter $\alpha$, calculated with the $\operatorname{\pi iC}$ approximations $\operatorname{\pi C}\left(C_{2}\right), \operatorname{\pi c}\left(C_{2}^{(2)}\right)$, $\operatorname{\pi C}\left(C_{2}+C_{3}^{\mathrm{S}}\right), \operatorname{TIC}\left(C_{2}^{(2)}+C_{3}^{\mathrm{S}}\right)$ and $\operatorname{Tic}\left(C_{2}^{(2)}+C_{3}^{\mathrm{F}}\right)$. The strip delimited by broken lines is the $2 \sigma$ confidence interval for the DMC energy.

In figure 1 we show the dependence of various TIC approximations on the variational HO length parameter $\alpha$ of (16). If the wavefunction were exact, there would be no dependence at all. Not surprisingly, the simple $\operatorname{TIC}\left(C_{2}\right)$ wavefunction shows a strong dependence, but 
it is gratifying to see that at the much more complete $\operatorname{TIC}\left(C_{2}^{(2)}+C_{3}^{\mathrm{F}}\right)$ level this dependence has almost disappeared. We are thus led to believe that not only the energy eigenvalue, but also the wavefunction itself is well represented by this parametrization. The data presented in section 4.2 below reinforce this belief.

That the energy values are good is evident from comparison with the DMC results. This is also seen from table 3 , where various TIC results for the ${ }^{4} \mathrm{He}$ ground-state energy with the MT-V potential are compared with those obtained with other methods.

Table 3. Comparison of the nc method with other calculational methods for the ground-state energy (in MeV) of the ${ }^{4} \mathrm{He}$ nucleus with the 33 and MT-V potentials. Shown are the results for various TIC methods described in the text, hyperspherical harmonics (HH) description in the potential basis with pair correlations (PPB) [30,32], Jastrow correlated HH (CPB) [34], the integro-differential equation approach to the HH expansion (DEA) [33], the ATMS method [27]. the coupled rearnangement of channels (CRC) [35] method, the coupled-cluster method (CCM) at both Sub(3) and Sub(4) levels of approximation [17], the Fadeev-Yakubovsky (FY) method [36] and the diffusion Monte Carlo (DMC) method [28].

\begin{tabular}{lll}
\hline & \multicolumn{2}{c}{ Potential } \\
\cline { 2 - 3 } Method & MTV & s3 \\
\hline PPB & -30.05 & -26.005 \\
IDEA & -30.68 & -27.09 \\
CPB & -31.344 & -27.369 \\
ATMS & -31.36 & - \\
CRC & -31.357 & - \\
CCM Sub(3) & -31.24 & - \\
$\propto C M ~ S u b(4)$ & -31.36 & - \\
FY & -31.36 & - \\
$\operatorname{TTC}\left(C_{2}\right)$ & -29.41 & -25.37 \\
$\operatorname{TC}\left(C_{2}+C_{3}^{5}\right)$ & -30.71 & -26.67 \\
$\operatorname{TC}\left(C_{2}+C_{3}^{5}+C_{4}^{S}\right)$ & -30.72 & - \\
$\operatorname{TCC}\left(C_{2}^{(2)}+C_{3}^{\mathrm{F}}\right)$ & -31.24 & -27.21 \\
$\mathrm{DMC}$ & $-31.32 \pm 0.02$ & $-27.35 \pm 0.02$ \\
\hline
\end{tabular}

The methods based on the hyperspherical harmonics (HH) expansion are most similar to our own. Given that the HH basis is complete, one may obtain an exact representation of the wavefunction in terms of it [29], as well as in terms of any other complete basis such as the shell-model basis. The convergence of the HH expansion is, however, very slow, so that it is preferable to use an alternative such as the pair-correlated form, which is a generalization of the Fadeev representation for the three-body problem. It corresponds to a wavefunction of the form

$$
\Psi=\sum_{i<j} g\left(r_{i j}, h_{4}\right)
$$

where, as before, $h_{4}$ is the four-particle hyper-radius. The pair-correlated potential basis (PPB) method is an approximation to this wavefunction, and corresponds to considering only a selected set of hyperspherical harmonics when expanding (17) [30-32], specifically those related to the two-body potential. The zeroth-order term of this expansion almost coincides with our $\operatorname{TIC}\left(C_{2}\right)$ approximation. The higher terms produce a small, but non-negligible, 
correction to the ground-state energy of about $0.5 \mathrm{MeV}$, as can be seen from table 3 . As can also be seen from table 3 , the accuracies obtained via the PPB and $\operatorname{TrC}\left(C_{2}\right)$ methods are in fact quite similar. The TIC calculation that most closely resembles the PPB one is TIC $\left(C_{2}+C_{3}^{\mathrm{s}}+C_{4}^{\mathrm{S}}\right)$. This actually performs considerably better than the PPB method for the MT-V potential, presumably due to the full optimization done on the true pair correlation function.

The integro-differential equation approach (IDEA) [33] is itself an approximation to the PPB method, in which the infinite number of coupled integral equations are replaced by a pair of integro-differential equations coupling the pair correlation and hyper-radiusdependent parts. Although this is technically much simpler than the PPB method, the upperboundedness character of the resulting eigenvalue is lost. Interestingly, this produces an energy very close to that of the $\operatorname{TIC}\left(C_{2}+C_{3}^{\mathrm{S}}+C_{4}^{\mathrm{S}}\right)$ method.

The PPB is not a complete basis. The natural way of improving the PPB method is to include other harmonics not contained in the potential basis. However, it has been found to be more practical to change the basic description by substituting a Jastrow-correlated ansatz for the pair-correlated ansatz. The wavefunction is then described as a product of a Jastrow factor and a function to be expanded in the HH basis. When this latter expansion is limited to the potential basis the method is called the correlated potential basis (CPB) approximation [34]. The correlation is determined by solving a screened Schrödinger equation for a pair of particles. The optimization effort is concentrated on the HH expansion. Not surprisingly, this wavefunction is capable of generating essentially all of the energy for this system, though it must be added that this method produces a far from simple calculation.

We also show in table 3 the results from three other analytical methods, each of which is in principle exact. These are the ATMS [27] and the coupled rearrangement of channels (CRC) [35] methods, both of which are based on expansions over the two-body channels, and the CCM [17], which is closest in spirit to the present method. Finally, the fully converged result recently obtained from the solution of the Fadeev-Yakubovsky equation [36] is also quoted.

Our own TIC results are obtained by solving for the linear variational parameters in, for example, (12), as eigenvector coefficients in a generalized eigenvalue equation. As a byproduct we also produce, at no additional cost, values for the breathing-mode excited states. For ${ }^{4} \mathrm{He}$ with the $\mathrm{MT}-\mathrm{V}$ potential, we obtain via the $\mathrm{TIC}\left(C_{2}^{(2)}+C_{3}^{\mathrm{F}}\right)$ method values of $-30.67,-5.71$ and $9.54 \mathrm{MeV}$ for the three lowest energy values. The excitation energy of the first breathing mode state is close to the value of $22.86 \mathrm{MeV}$ obtained by the CRC method [35]. The ground-state energy here is somewhat higher than the value quoted in table 2 because we have now minimized with respect to the first breathing-mode energy.

\subsection{Wavefunction properties}

That the TIC method produces excellent energy values is beyond question. In addition, there is strong evidence that the wavefunction itself is accurately represented. Once again, the structure of the TIC wavefunction makes it both straightforward and efficient to use for quantities other than the energy. If expressed using Gaussian expansions, the $N$-body bosonic TIC wavefunction can be written in the very general analytic form

$$
\Psi_{\mathrm{TIC}}=\left(\frac{\alpha}{\sqrt{\pi}}\right)^{6} \exp \left(-\frac{1}{2} \alpha^{2} \sum_{i}^{N} r_{i}^{2}\right) \sum_{n} c_{n} \exp \left(\sum_{i<j}^{N} \beta_{i j}^{(n)} r_{i j}^{2}\right)
$$

where the linear coefficients $\left\{c_{n}\right\}$ can be easily chosen to normalize the wavefunction. 


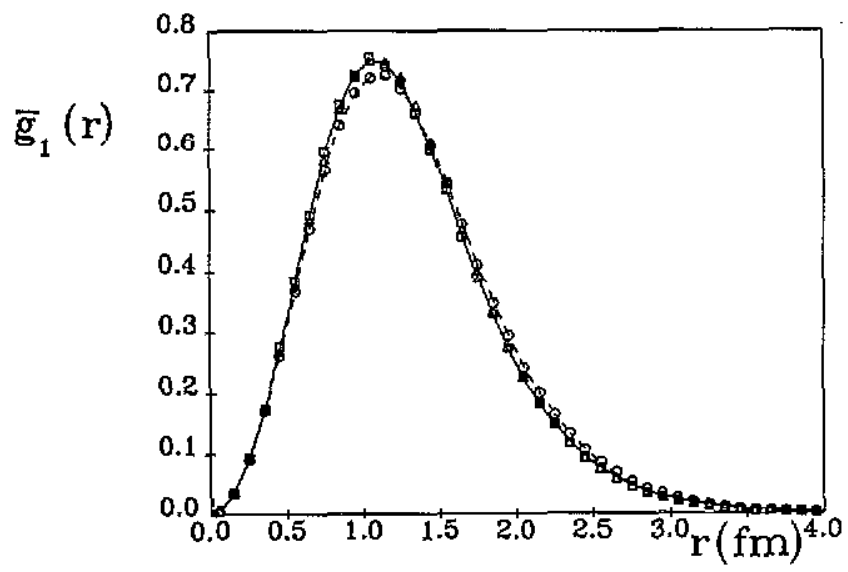

Figure 2. One-body distribution function $\bar{g}_{1}(r)$ for the ground-state ${ }^{4} \mathrm{He}$ nucleus described by the MT-v potential. Results shown are for diffusion Monte Carlo (full curve), the mc( $C_{2}$ ) (broken curve), $\pi \mathrm{C}\left(C_{2}^{(2)}\right)$ (circles), $\pi \mathrm{C}\left(C_{2}^{(2)}+C_{3}^{\mathrm{S}}\right.$ ) (triangles) and $\operatorname{\pi c}\left(C_{2}^{(2)}+C_{3}^{\mathrm{F}}\right)$ (squares) approximations.

Once we have the wavefunction in this normalized form, calculation of wavefunction properties is straightforward. The one-body distribution function

$$
\begin{aligned}
& g_{1}(r) \equiv \int \mathrm{d} r_{1} \cdots \int \mathrm{d} r_{N}\left|\Psi\left(r_{1}, \ldots, r_{N}\right)\right|^{2} \delta\left(r+R-r_{1}\right) \\
& \bar{g}_{1}(r) \equiv 4 \pi r^{2} g_{1}(r)
\end{aligned}
$$

is the most complicated object we calculate, because the delta function does not allow the integral to be decomposed into intrinsic and centre-of-mass pieces. Nonetheless, the analytic expression for the wavefunction does allow a simple stochastic evaluation of this quantity, with minimal error. Once the function $\overline{g_{1}}(r)$ is calculated, the mean square radius

$$
\left\langle r^{2}\right\rangle=\int \mathrm{d} r r^{2} g_{1}(r)=\int_{0}^{\infty} \mathrm{d} r r^{2} \bar{g}_{1}(r)
$$

is trivially obtained. It is perhaps worth mentioning that this same expectation value can also be obtained from the much simpler distribution function which is analytically calculable

$$
\hat{g}_{1}(r) \equiv \int \mathrm{d} r_{1} \cdots \int \mathrm{d} r_{N}\left|\Psi\left(r_{\mathrm{t}}, \cdots, r_{N}\right)\right|^{2} \delta\left(r-r_{1}\right)
$$

via the simple relation

$$
\left\langle r^{2}\right\rangle=\int \mathrm{d} r r^{2} \hat{g}_{1}(r)-\int \mathrm{d} R R^{2} \Phi_{\mathrm{CM}}^{2}(R)\left(\int \mathrm{d} R \Phi_{\mathrm{CM}}^{2}(R)\right)^{-1}
$$

even though $g_{1}(r)$ and $\hat{g}_{1}(r)$ are themselves not obviously related.

The two-body distribution function

$$
\begin{aligned}
& g_{2}(r) \equiv \int \mathrm{d} \boldsymbol{r}_{1} \cdots \int \mathrm{d} r_{N}\left|\Psi\left(r_{1}, \cdots, r_{N}\right)\right|^{2} \delta\left(r-\left(r_{1}-r_{2}\right)\right) \\
& \overline{g_{2}(r)} \equiv 4 \pi r^{2} g_{2}(r)
\end{aligned}
$$

is also analytically calculable. 
In figure 2 we show the one-body distribution function $\overline{g_{1}}(r)$ for the ground-state ${ }^{4} \mathrm{He}$ nucleus using the MT- $\vee$ potential, calculated via the diffusion Monte Carlo method (mixed estimator), and various TIC approximations. (Note that the functions shown satisfy the normalization condition implied by (19), namely $\left.\int_{0}^{\infty} \overline{g_{1}}(r) \mathrm{d} r=1\right)$. The errors due to the Monte Carlo evaluation of $\overline{g_{1}}$ are at most 0.013 , occurring for small $r$ values where the statistics are worst. The agreement with the DMC results is essentially complete at the highest level of approximation. Once again, we see how well the $\operatorname{TIC}\left(C_{2}^{(2)}+C_{3}^{S}\right)$ approximation does, even at the wavefunction level.

Table 4. The RMS radius of the ${ }^{4} \mathrm{He}$ nucleus, described by the MT-V potential, as calculated with various translationally invariant cluster $(\mathrm{nC})$ approximations, as well as with several other calculational methods, namely the coupled cluster method (CCM) [17] at various levels of truncation, the Green function Monte Carlo (GFMC) method [25], the ATMS [27] method, and the diffusion Monte Carlo (DMC) method [28].

\begin{tabular}{ll}
\hline Method & $\left\langle r^{2}\right\rangle^{1 / 2}(\mathrm{fm})$ \\
\hline $\operatorname{\pi IC}\left(C_{2}\right)$ & 1.43 \\
$\operatorname{\pi IC}\left(C_{2}^{(2)}\right)$ & 1.44 \\
$\operatorname{\pi C}\left(C_{2}^{(2)}+C_{3}^{S}\right)$ & 1.40 \\
$\operatorname{TC}\left(C_{2}^{(2)}+C_{3}^{\mathrm{F}}\right)$ & 1.41 \\
& \\
$\operatorname{CCM~Sub}(2)$ & 1.41 \\
$\operatorname{CCM~Sub(3)}$ & 1.36 \\
$\operatorname{CCM~Sub(4)}$ & 1.39 \\
ATMS & 1.40 \\
GFMC & $1.36 \pm 0.01$ \\
DMC & $1.395 \pm 0.005$ \\
\hline
\end{tabular}

In table 4 we present values of the point RMS radius for the ${ }^{4} \mathrm{He}$ nucleus for various TIC approximation levels (and calculated from the $\overline{g_{1}}(r)$ of figure 2), as well as those obtained with other methods. It is somewhat surprising that the $\operatorname{TIC}\left(C_{2}^{(2)}+C_{3}^{\mathrm{F}}\right)$ number is not in agreement with the CCM Sub(3) value (even though their energies are identical) but is in agreement with the CCM Sub(4) value.

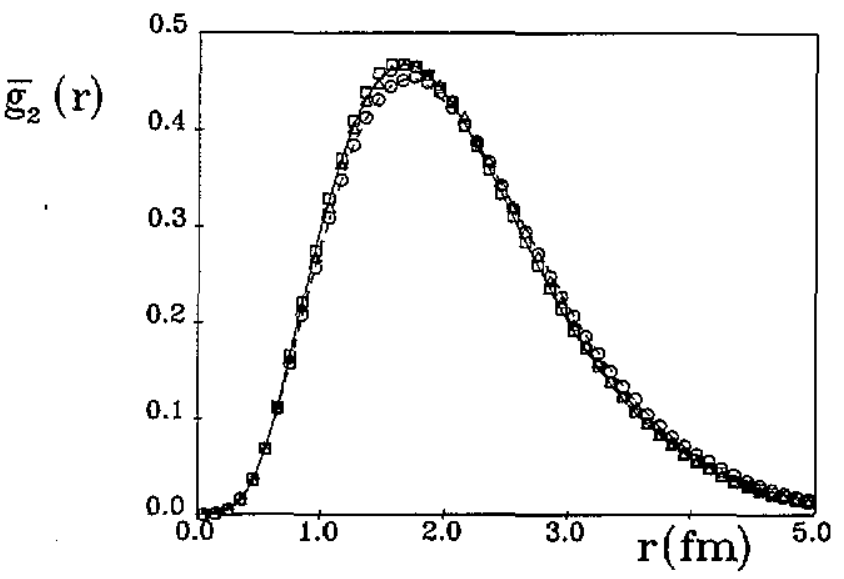

Figure 3. Two-body distribution function $\bar{g}_{2}(r)$, as per figure 2 . 


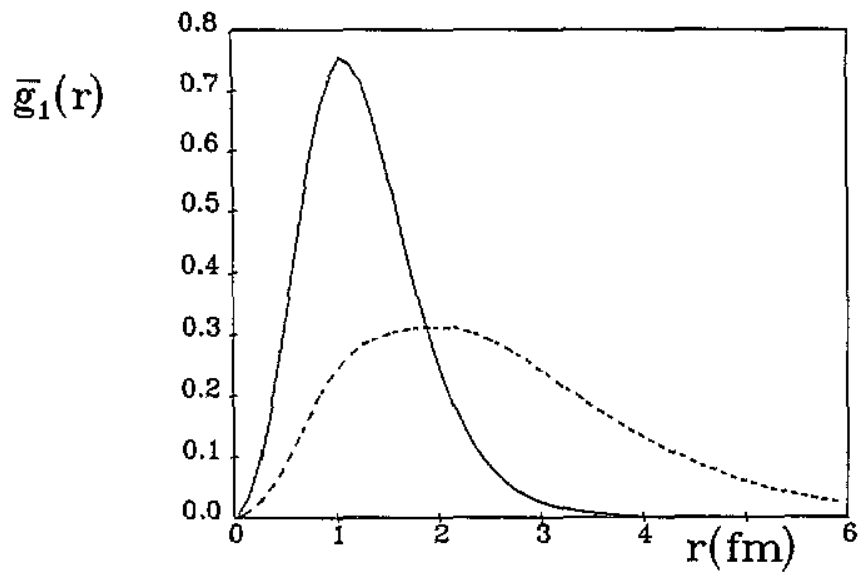

Figure 4. A comparison of the one-body distribution functions $\overline{g_{1}}(r)$ for the ${ }^{4} \mathrm{He}$ nucleus described by the MT-V potential, for the ground (full curve) and first excited breathing mode (dotted curve) states, both calculated in the $\operatorname{TIC}\left(C_{2}^{(2)}+C_{3}^{F}\right)$ approximation.

Results for the two-body distribution function $\overline{g_{2}}(r)$ for the same system and methods are presented in figure 3. Once again, we find very impressive agreement with the DMC data. Finally, as mentioned above, the TIC method also readily produces results for the breathing-mode excited states. Figure 4 compares the one-body distribution functions for the ground state and first breathing mode of the ${ }^{4} \mathrm{He}$ nucleus with the $\mathrm{MT}-\mathrm{V}$ potential.

It is also worth noting that the wavefunction of (3) does not impose positive-definiteness on the Bose ground state, as must hold for the exact ground state. All that we are guaranteed is that the variational method used will generate the lowest-energy value for the chosen form of the wavefunction. Of course, if we have a sequence of improved wavefunctions which describe the system with increasing accuracy, we expect this important property to be increasingly satisfied. This is exactly what we find with the TICM. Using a random sampling of the ${ }^{4} \mathrm{He}$ MTT-V wavefunction we find, for example, that the number of negative values drops from 10 out of 200000 for the $\operatorname{TIC}\left(C_{2}^{(2)}\right)$ method to 0 out of 50000 for the $\operatorname{TIC}\left(C_{2}^{(2)}+C_{3}^{F}\right)$ method.

\section{Discussion}

This work began with the aim to reexamine the centre-of-mass problem in the context of applications of the coupled cluster method to light nuclei [1]. The direct result of this was to show how the CCM, without great changes in the usual formalism, could be modified to eliminate the problem of the spurious $\mathrm{CM}$ motion, by restricting from the outset the excitations produced to those which explicitly do not excite the CM. It was also shown that this could only be done if the usual $n \mathrm{p}-n \mathrm{~h}$ excitations used to build up the CCM wavefunction could no longer operate in isolation.

In later work, the entire approach was recast directly into coordinate space [3], and this led to the method presented in this work, namely the translationally invariant cluster method. This builds up excitations by acting on some model state by a sum of $n$-body cluster functions which are independent from one another, in the sense that they are not 
restricted to produce separate sets of excitations. Thus, for example, the three-body cluster function will produce correlations of a three-body nature, as well as of a two-body nature.

When joined with the Gaussian expansion method for the coordinate-space correlation functions, the resulting method is both flexible and expandable as well as computationally efficient. Since the method is strictly variational, the resulting approximation for the groundstate energy at any level of implementation is always an upper bound. The same is also true for the breathing-mode excited states. Furthermore, since the $\mathrm{CM}$ motion problem simply does not arise, the method is ideally suited to few-body systems. In applications shown in this paper to bosonic nuclei, the method is shown to be capable of generating energy values with accuracies comparable to any other many- or few-body calculational scheme. More importantly, the point-mass RMS radius data shown indicate that the wavefunction itself is well represented Preliminary results for fermionic systems, using central spin- and isospin-dependent forces, lead us to expect equal success with these more realistic systems. Nevertheless, calculations with realistic forces including tensor-, spin-orbit- and quadratic spin-orbit-dependent terms still require a considerable amount of algebraic work with the Tic method.

\section{Acknowledgments}

This work was developed under an Acciones Integradas research programme between Spain and the United Kingdom. EB and RG are supported by the Comision Interministerial de Ciencia y Tecnología, Spain. MFF acknowledges the support of a fellowship from the Ministerio de Educación y Ciencia, Spain, under the program Estancias Temporales de Científicos y Tecnólogos Extranjeros. RFB acknowledges the support of a research grant from the Science and Engineering Research Council of Great Britain.

\section{References}

[1] Bishop R F, Flynn M F, Bosca M C, Buendfa E and Guardiola R 1990 Phys. Rev. C 421341

[2] Bishop R F, Flynn M F, Bosca M C, Buendia E and Guardiola R 1990 The Nuclear Equation of State, A ed W Greiner and H Stöcker (New York: Plenum) p 605

[3] Bishop R F, Flynn M F, Buendía E, Boscá M C and Guardiola R 1990 J. Phys. G: Nucl. Part. Phys. 16 L61

[4] Bishop R F, Fiynn M F, Boscá M C, Buendía E and Guardiola R 1990 Condensed Matter Theories 5 ed $\checkmark$ C Aguilera-Navarro (New York: Plenum) p 253

[5] Bishop R F, Buendfa E, Flynn M F and Guardiola R 1991 J. Phys. G: Nucl. Part. Phys. 17857

[6] Bishop R F, Buendía E, Flynn M F and Guardiola R 1991 Condensed Matter Theories 6 ed S Fantoni and $S$ Rosati (New York: Plenum) p 405

[7] Bishop R F, Buendía E, Flynn M F and Guardiola R 1992 J. Phys. G: Nucl. Part. Phys. 181157

[8] Ceuleneer R, Semay C and Vandepeutte P 1990 J. Phys. G: Nucl. Part. Phys. 16 L295

[9] Boys S F 1950 Proc. R. Soc. A 200542

[10] Wilson K G 1990 Nucl, Phys. B (Proc. Suppl.) 1782

[11] Roothaan C C J 1951 Rev. Mod. Phys. 2369

Hall G G 1951 Proc. R. Soc. A 205541

[12] Boys S F 1960 Proc. R. Soc. A 258402

[13] Singer K 1960 Proc. R. Soc. A 258412

[14] Longstaff J V and Singer K 1960 Proc. R. Soc. A 258421

[15] Szalewicz K, Jeziorski B, Monkhorst H J and Zabolitzky J G 1983 J. Chem. Phys. 781400

[16] Szalewicz K, Jeziorski B, Monkhorst H J and Zabolitzky J G 1983 J. Chem. Phys. 795543

[17] Zabolitzky J G 1981 Phys. Lett, 100B 5

[18] Fink M 1974 Nucl. Phys. A 221163

[19] Fabre de la Ripelle M, Fiedeldey H and Sofianos A 1988 Phys. Rev. C 38449 
[20] Malfliet R A and Tjon J A 1969 Nuct. Phys. A 127161

[21] Afnan I R and Tang Y C 1968 Phys. Rev. 1751337

[22] Volkov A B 1965 Nucl. Phys. A 7433

[23] Brink D M and Boeker E 1967 Nucl. Phys. A 911

[24] Friar J L, Gibson B F and Payne G L 1982 Phys. Rev. C 252279

[25] Zabolitzky J G and Kalos M H 1981 Nucl. Phys. A 356114

[26] Carlson J, Pandharipande V R and Wiringa R B 1983 Nucl. Phys. A 40159

[27] Akaishi Y 1984 Nucl. Phys. A 416409

[28] Bishop R F, Buend\}a E, Flyn M F and Guardiola R 1992 J. Phys. G: Nucl. Part. Phys. 18 L21

[29] Fabre de la Ripelle M and Navarro J 1979 Ann. Phys., NY 123185

[30] Ballot J L 1981 Z. Phys. A 302347

[31] Ballot J L, Fabre de la Ripelle M and Navarro J 1984 Phys. Lett. 143B 19

[32] Kievsky A, Viviani M and Rosati S 1991 Condensed Matter Theories 6 ed S Fantoni and S Rosati (New York: Plenum) p 391

[33] Oehn W, Sofianos S A, Fiedeldey H and Fabre de la Ripelle M 1990 Phys. Rev. C 422322

[34] Viviani M, Kievsky A and Rosati S 1992 Nuovo Cim. A 1051473

[35] Kameyama H, Kamimura M and Fukushima Y 1989 Phys. Rev. C 40974

[36] Kamada H and Glöckle W 1992 Nucl. Phys. A 548205 